\title{
Les profils LinkedIn de salariés, une activité bénévole au service de l'entreprise ? \\ L'AVE-In (Activités de valorisation de l'employeur sur LinkedIn) et problématique du Digital Labor
}

\author{
Hélène Hoblingre KLEIN
}

\begin{abstract}
Résumé :
Les activités de valorisation de l'employeur sur LinkedIn (AVE-In) déployées par les salariés d'entreprise constituent un nouveau champ de recherche. A la lisière entre utilisation interne et externe l'AVE-In s'avère difficile à catégoriser. Cet article présente les résultats d'un questionnaire qui vise à mieux comprendre les caractéristiques de l'AVE-In propres au travail, au loisir et au bénévolat. Les résultats de l'étude mettent en évidence des correspondances entre l'AVE-In et le bénévolat classique, le bénévolat marginal et le bénévolat d'entreprise. L'hypothèse selon laquelle l'AVE-In se situerait à la lisière entre ces deux dernières formes de bénévolat et constituerait une extension bénévole de l'activité salariée reste encore valide mais non encore confirmée.
\end{abstract}

\section{Introduction}

La valorisation des actes bénévoles sur le Web trouve ses racines dans les activités des pionniers d'Internet (Cardon \& Casilli, 2015, 58). Ces derniers, dont les contributions étaient indépendantes de toute volonté de rémunération, ont hissé au premier rang leurs valeurs de partage. Ce sous-bassement idéologique s'est matérialisé dans divers projets bénévoles tels que Wikipédia. L'engagement bénévole s'est ensuite quelque peu monétarisé au travers des entreprises du web 2.0 qui ont passé implicitement avec les internautes l'accord suivant : "à vous l'enrichissement personnel lié aux possibilités de communication et de partage en ligne, à nous l'enrichissement financier lié à la monétisation de ces activités » (Broca, 2016). Ce basculement, d'un bénévolat dégagé de toute perspective de profit financier à un bénévolat indirectement monétarisé, soulève la question de la nature des nouvelles activités déployées gratuitement sur le Web, mais faisant cependant l'objet d'un calcul économique de la part d'instances pouvant en tirer financièrement profit. Le concept de Digital Labor, défini comme une « activité de faible intensité (assimilable à du loisir) et requérant une faible expertise tout en produisant une forte valeur au bénéfice du propriétaire de la plateforme numérique » (Pierre \& Alloing, 2015), met en exergue la difficulté qu'il y a aujourd'hui à faire la distinction sur internet entre le don et le calcul (Cardon, 2015). Enfin, dans un ouvrage sur les communautés de pratique, nous avons démontré que LinkedIn était le lieu de pratiques participatives orientées vers l'intérêt de communauté, dans une perspective de codéveloppement (Hoblingre Klein \& Audran, 2017). Or, comme le souligne Mercier, le «concept de codéveloppement est un des fondements du bénévolat et de la bienfaisance, du coopératisme et de la coconstruction de corpus de connaissances » (Mercier \& Plaisent, 2010, 168). Cependant, il est également apparu que l'activité en réseau sur LinkedIn pouvait être tendue entre intérêt personnel et intérêt de communauté (Hoblingre Klein \& Audran, 2017).

Appréhender les activités qui se situent à la lisière entre partage libre et exploitation économique, entre intérêt personnel et intérêt de communauté apparaît alors comme un nouveau champ de recherche, pour lequel les réseaux sociaux numériques (RSN) offrent un précieux terrain d'investigation. Larroche \& Piment, (2014) mettent ainsi en avant les tensions qui existent entre praxis (expression de soi) et poiésis (action productive). Ils soulignent que si les conversations qui se déroulent sur Facebook sont exercées dans un contexte professionnel, leur reconnaissance en tant que métier est soumise à l'intégration d'une activité productive (poiésis) à la praxix. D’ailleurs, Louis-Pierre Guillaume révèle que les dirigeants d'entreprise sont de plus en plus nombreux à investir dans les réseaux sociaux dont ils reconnaissent les bénéfices en termes de productivité (Guillaume, 2017). De plus, à l'instar des autres réseaux sociaux professionnels tels que Viadeo (Povéda, 2016), LinkedIn est l'objet d'importants enjeux réputationnels. Or, comme le souligne Louise Merzeau, «les situations relevant de l'e-réputation ont en commun de mettre en œuvre une récursivité qui enchâsse les individus, communautés et firmes dans une circularité d'influences et de calculs » (Merzeau, 2015, 20). Enfin, on observe que l'utilisation de LinkedIn (leader mondial des réseaux sociaux numériques 
professionnels) par les acteurs des ressources humaines implique de concilier deux facettes «l'une sous la marque d'un engagement collectif, l'autre centrée sur l'individu » (Hoblingre et al., 2016, 168).

Ces différents constats nous amènent à nous demander si le bénévolat peut constituer une catégorisation intéressante pour appréhender ces activités, à la fois internes et externes, qui se situent à la lisière entre exposition de soi et promotion d'une entreprise, entre gratuité et calcul. Cet article tente de répondre à cette question en s'appuyant principalement sur un questionnaire adressé à 117 personnes. Une enquête basée sur des entretiens est venue confirmer certains éléments de réponse.

Une précédente recherche nous a déjà permis de démontrer que de nombreux consultants RH et recruteurs valorisent via leur profil LinkedIn l'activité et l'image de leur employeur. Tout d'abord, certains d'entre eux intègrent dans la partie « résumé » de leur profil un texte descriptif leur permettant de mettre en avant l'activité de leur employeur. Souvent, des liens hypertextes redirigent le visiteur vers le site de l'entreprise. D'autres encore intègrent des accroches destinées à favoriser leur travail de prospection commerciale ou de recrutement. Des phrases telles que «nous sommes à la recherche de nouveaux talents [...] vous cherchez un poste de développeur ? Envoyez-nous votre candidature !» sont alors utilisées. Enfin, certains salariés rédigent sur leur profil des "posts », c'est-à-dire des messages relativement courts donnant de rapides informations sur des actualités relatives à l'entreprise pour laquelle ils travaillent. Ces trois types d'activités déployées par le biais des profils LinkedIn (valorisation de l'image de l'employeur sur le profil, utilisation du profil comme outil de travail, publication de "posts » relatifs aux actualités de l'employeur) seront désignées dans cet article sous la forme du sigle AVE-In (Activités de valorisation de l'employeur sur LinkedIn).

Parfois imposée, parfois suggérée ou choisie indépendamment de tout discours extérieur, l'AVE-In ne se laisse pas aisément catégoriser : s'agit-il d'un travail salarié, d'un loisir, d'un engagement bénévole, voire d'une extension bénévole de l'activité salariée assimilable au Digital Labor, puisque ce dernier reflète « une vision où l'ethos du don complète dans un équilibre instable les éléments utilitaires ou d'intérêt économique » (Cardon \& Casilli, 2015, 15) ? Cette contribution qui se situe dans le champ de l'étude des transformations professionnelles induites par l'irruption des outils de communication numérique apportera quelques pistes de réponse. En nous appuyant sur des notions issues de la sociologie du travail et sur les recherches relatives aux nouvelles technologies de communication, nous présentons les résultats d'une enquête qualitative menée en 2017 et visant à établir des recoupement entre l'AVE-In et les caractéristiques du bénévolat. Il s'agit également de savoir si, avec le développement des nouvelles technologies, l'activité se déployant «dans une zone qui se situe en lisière (en marge même pour certains) de leur fonction professionnelle statutaire » (Audran, 2002, 99) est du bénévolat.

Nous exposons dans une première partie le contexte général dans lequel s’inscrit cette étude : la mutation du travail à l'aire numérique. Dans une deuxième partie, nous analysons les similitudes qui existent entre l'AVE-In et certaines caractéristiques définitionnelles du travail, du loisir et du bénévolat. Nos différents constats nous permettent d'élaborer une méthodologie que nous détaillons dans une troisième partie. Enfin, les données issues de notre enquête sont présentées puis débattues dans une quatrième et dernière partie.

\section{La mutation du travail à l'aire numérique}

La révolution numérique, et plus particulièrement l'essor du web 2.0 dans les années 2000 (Vial, 2013), a considérablement transformé le travail, si bien que neuf nouvelles formes d'emploi sont récemment apparues (Valenduc \& Vendramin, 2016, 33). L'analyse de certaines de ces formes nous révèle les mutations du travail en termes de lieu, de temporalité et de tâches.

Au niveau spatio-temporel, l'arrivée du télétravail, c'est-à-dire du travail a distance, a permis de déplacer le lieu traditionnel d'exercice de l'activité mais également de transformer les horaires habituels de travail. Il est désormais possible de travailler la nuit depuis chez soi, ou même depuis un train, un hôtel, un café... Ce bouleversement spatio-temporel a remis en question la frontière entre la sphère privée et professionnelle (Metzger \& Cléach, 2004). Cette porosité semble propice à la confusion entre les engagements professionnels et personnels et donc entre le salariat et le bénévolat. Les nomades numériques, qui s'apparentent à des télétravailleurs n'ont, quant à eux, aucune localisation fixe. Il en découle que leur rémunération est assujettie aux résultats et qu'ils doivent faire preuve d'une «disponibilité potentielle permanente » (Valenduc \& Vendramin, 2016, 34). On comprend donc ici que la mutation du travail au niveau spatial et temporel favorise un engagement supplémentaire et presque inconditionnel du travailleur. 
En ce qui concerne les tâches, le numérique a permis «l'instrumentalisation de nos sociabilités numériques ", c'est-à-dire que nos traces identitaires numériques ont acquis une dimension marchande (Ertzscheid, 2013) par ailleurs sous-estimée par les internautes. Des activités à but non lucratif tournées vers la collectivité peuvent ainsi être réinvesties par des tiers dans une perspective financière. Saisir des données sur internet, notamment à l'occasion d'un achat (Dujarier, 2014), constitue alors un travail qui ne dit pas son nom ... L'économie numérique a également fait émerger un autre type de travail aux finalités économiques plus explicites : le crowdwording, littéralement «travail de la foule ». Le crowdworking a été défini comme le fait d'externaliser auprès d'un très large groupe d'individus un travail traditionnellement réalisé par un employé (Felstiner, 2011). Pour cette « externalisation ouverte du travail » (Valenduc \& Vendramin, 2016, 33) des plateformes telles qu'Amazon font appel à des travailleurs peu qualifiés tandis que d'autres, telles que freelance.com, requièrent des individus possédant des qualifications spécifiques (Baudry \& Chassagnon, 2017). Ce phénomène est révélateur de l'essor de l'économie collaborative et peut ainsi être rapproché de l'uberisation du travail : par l'entremise de plateformes numériques, il est désormais possible, et pour une palette de plus en plus large d'activités, de répondre à des offres de projet ou de réaliser des tâches précises à la demande de clients.

\section{L'AVE-In : entre travail, loisir et bénévolat}

Dans ce paysage particulier, nous reconnaissons que la distinction entre le travail, le loisir et le bénévolat reste discutable tant ces catégories s'entremêlent les unes aux autres. La distinction travail-loisir est sans cesse remise en question par les nouvelles technologies numériques qui ont «favorisé la création de catégories entièrement nouvelles de travail 'digital' ou 'virtuel', à la fois rémunéré et non rémunéré » (Vendramin et al., 2016, 9). Par ailleurs, il semblerait que l'action bénévole, même lorsqu'elle est vécue comme un loisir, représente un temps de travail à part entière en raison essentiellement de la nécessaire mobilisation de compétences. En effet, «l'expérience antérieure d'un métier, d'une pratique professionnelle ou d'un diplôme sont des prérequis dans plusieurs domaines du bénévolat » (Kesteman \& Monnier, 2005, 56). Enfin, l'économie collaborative induite par le numérique repose sur «le partage ou l'échange de différents biens, services ou connaissances « avec échange monétaire (vente, location, prestation de service) ou sans échange monétaire (dons, troc, volontariat) »1. Ainsi, on peut se demander si l'économie collaborative n'incarne pas un nouveau modèle socio-économique où le salariat et le bénévolat tendraient à être aisément confondus.

Malgré leur porosité, ces trois catégories nous sont apparues comme la grille d'analyse la plus pertinente pour l'élaboration d'une méthodologie de recherche. En effet, au travers notamment d'une première enquête réalisée en 2015-2016 nous avons identifié des similitudes entre l'AVE-In et certaines caractéristiques définitionnelles du travail, du loisir et du bénévolat.

\subsection{Travail}

De l'avis d'Yves Schwartz, «définir le travail est une tâche vaine » (Schwartz, 2010, 99). Les quatre caractéristiques propres au travail que nous avons retenues ici ne sauraient donc être restrictives, mais elles présentent pour intérêt de synthétiser différentes perspectives disciplinaires. D’un point de vue ergologique, l'individu au travail fait face à des débats de normes (Schwartz, 2010) et à des prescriptions qui le précèdent et entre lesquelles il doit arbitrer. Or, comme l'ont montré Hoblingre, Audran et Chevry, certains utilisateurs s'interrogent sur le sens qu'ils souhaitent donner à leurs actions sur LinkedIn par rapport à leur employeur (Hoblingre et al. 2016). Ils tentent alors de dénouer ou de dépasser la dichotomie entre l'utilisation personnelle et l'utilisation institutionnalisée de leurs profil (Hoblingre et Audran, 2017), ce qui n'est pas sans rappeler les débats de normes au sujet desquels Schwartz écrit, en soulignant les allers-retours incessants du travailleur entre niveau micro et niveau macro :

"Une situation de travail contient des enjeux de société. Inversement, par la fason dont il travaille, chacun prend position dans ces débats de société et les recompose à son échelle » (Schwartz \& Durrive, 2003, 14)

La psychodynamique du travail nous révèle que le travail constitue une confrontation au réel qui résiste toujours à la maîtrise (Dejours, 2003). Au niveau économique, le travail est avant tout producteur de valeur

1. Source : http://www.vie-publique.fr/actualite/dossier/rub1859/economie-collaborative-nouveau-modele-socioeconomique.html 
(Cardon \& Casilli, 2015) notamment sociale ou économique : il est utile et utilisé. Enfin, d'un point de vue sociologique, voire anthropologique, les relations de travail sont sous-tendues par des logiques de don contre-don (Jacquot, 2012). Ces trois caractéristiques sont encore une fois palpables dans les activités d'AVE-In. D'une part, l'utilisateur qui souhaite rendre visibles ses activités est constamment obligé de rappeler sa présence aux autres par une activité continue, au risque de se retrouver noyé dans la rapidité du flux d'information et l'abondance des profils. C'est notamment le cas sur Facebook où «il s'agit de se manifester sans cesse pour continuer d'exister et maintenir son réseau social» (Georges, 2009, 191). En ce sens, l'activité d'AVE-In peut représenter une confrontation aux exigences de visibilité d'un réseau résistant à la maitrise. Par ailleurs, les avantages économiques potentiels de l'AVE-In (promotion de l'image de marque de l'employeur et attraction de nouveaux clients ou de nouveaux salariés) ramènent cette activité à une perspective utilitaire.

\subsection{Loisir libre ou activité sous influence?}

Contrairement au travailleur, l'individu qui s'adonne au loisir agit en dehors de toutes considérations utilitaires. Et pour cause, le loisir est marqué par la notion de motivation intrinsèque (Riverin \& Simard, 2003) : l'individu s'investit dans le loisir en raison de l'intérêt même qu'il accorde à l'activité et du plaisir qu'elle lui procure. L'activité devient un but en soi. Le loisir est alors synonyme de liberté : il s'exerce librement et sans contrainte. D'ailleurs, on observe que c'est le caractère obligatoire d'une activité qui lui fait quitter le champ des loisirs (Riverin \& Simard, 2003) à l'instar des footballeurs professionnels ! En revanche, on distingue chez l'individu au travail des facteurs de motivation extrinsèques, tels que la rémunération et la collaboration, et des facteurs de motivation intrinsèques tels que l'accomplissement et les attributs de l'emploi (Zedini et al., 2016). La motivation apparait comme un indicateur important permettant d'une part, d'évaluer si une activité relève du loisir ou du travail, et, d'autre part, d'estimer le niveau de contrainte ou de liberté de l'individu.

De ce point de vue, l'AVE-In constitue une activité ambiguë ou du moins variable puisqu'elle peut être libre, contrainte ou suggérée. Cette ambiguité est palpable du côté des salariés comme de celui des entreprises. En effet, il est difficile d'appréhender dans quelles perspectives l'utilisateur pratique l'AVE-In : est-il animé par un élan d'accomplissement, pratique-t-il cette activité pour le plaisir qu'elle lui procure (motivation intrinsèque) ou agit-il pour percevoir un gain financier et dans une perspective de collaboration (motivation extrinsèque) ? Côté entreprises, on trouve des messages d'incitations pour favoriser l'utilisation de LinkedIn par leurs salariés, sans pour autant les obliger. Les entreprises ont en effet tendance à développer "une marque-employeur 2.0, caractérisée par une communication plus dialogique impliquant des salariés » (Larroche, 2014, 53). Le groupe américain Y de plus de 70000 salariés a fait parvenir en 2016 un document à ses employés pour que ces derniers veillent à la bonne présentation de leurs profils LinkedIn dans une perspective de valorisation réciproque :

En prenant des mesures pour améliorer votre profil LinkedIn, vous aurez. l'occasion de vous présenter comme un contributeur précieux dans votre domaine d'expertise et comme un ambassadeur de la marque Y. Ce faisant, vous renforcerez votre réputation dans votre domaine d'expertise tout en attirant des talents potentiels pour $Y$. Voici quelques Conseils pour vous aider à tirer le meilleur parti de LinkedIn (Groupe Y)2

Ce message fait visiblement appel à la fois à la motivation intrinsèque (s'imposer dans un domaine d'expertise relève d'une forme d'accomplissement) et à la motivation extrinsèque des salariés (être un ambassadeur et participer au recrutement se fait dans un esprit de collaboration).

Par ailleurs, on a observé il y a quelques années l'apparition de « networked entreprise » (Bughin \& Chui, 2010), des entreprises en réseautage qui utilisent abondamment le web 2.0 collaboratif pour mettre en relation les efforts produits en interne par les employés mais également pour trouver de nouveaux clients, partenaires ou fournisseurs. Enfin, une enquête menée auprès de 3249 cadres a révélé que deux tiers d'entre eux utilisent le web 2.0 dans leur entreprise et qu'un nombre croissant d'employés utilisent les technologies du web 2.0 dans le cadre de leur travail (ibid.). Sur cette base, il nous semble nécessaire de comprendre dans

2. Traduction de l'auteur: By taking steps to enhance your LinkedIn profile, you'll have the opportunity to present yourself as a valuable contributor within your area of expertise and $Y$ brand ambassador, strengthening your reputation within your area of expertise while attracting potential talent for Y. Here are some tips to help you get the most out of LinkedIn. (Groupe Y) 
quelles mesures l'AVE-In est vécu par ceux qui l'exercent comme une activité libre, contrainte ou sous influence, mais également d'appréhender les motivations de ces derniers.

\subsection{Bénévolat}

Nos recherches théoriques ont mis en exergue la pluralité et l'hétérogénéité des formes qu'emprunte le bénévolat. C'est donc au travers de ces différentes catégories de bénévolat que nous nous attacherons à dépeindre l'AVE-In.

\subsubsection{Bénévolat classique et marginal}

Une précédente enquête nous a permis de démontrer que LinkedIn est utilisé «pour partager de l'information au sein d'une communauté qui possède les mêmes intérêts » (Hoblingre \& Chevry Pébayle, 2016, 908). Sur cette base, on peut se demander si l'activité d'AVE-In n'est pas l'expression d'un élan de solidarité et de générosité, à l'instar du bénévolat que nous qualifierons ici de «classique ». Cette forme de bénévolat « exprimerait avant tout un désir d'engagement, un don de soi et un élan de générosité » (Simonet, 2010, 7-8). Ainsi, c'est avant tout la motivation intrinsèque qui préside l'acte bénévole (Bruna \& Damiano, 2009). Stebbins rapproche quant à lui le bénévolat classique du loisir en tant qu'« activité librement » choisie. (Stebbins, 1996, 212). Il souligne également que le bénévole est essentiellement motivé par la volonté d'aider les autres mais également de s'aider soi-même (ibid., p. 213).

Le choix constitue d'après nous un paramètre définitionnel clef, puisqu'il permet d'une part de dissocier le travail du bénévolat classique, mais également le bénévolat classique du bénévolat dit " marginal». Simonet oppose ainsi un bénévolat choisi à un bénévolat subi. D’après elle, il y aurait d'un côté « un vrai bénévolat qui reposerait, comme le sens commun le définit, sur le critère de libre choix et de bonne volonté » (Simonet, 2010, 54) et de l'autre « un bénévolat instrumentalisé et donc détourné ou dénaturé » (ibid., p. 54). C'est cette forme d'acte bénévole contraint que Robert Stebbins a initialement désigné sous le terme de «bénévolat marginal» (Stebbins, 1996, 221), expression ensuite reprise par Simonet. La question du caractère choisi ou non de l'AVE-In occupera donc une place primordiale dans notre enquête.

\subsubsection{Bénévolat d'entreprise}

On mentionnera enfin le bénévolat d'entreprise correspondant à la "promotion auprès des salariés d'activités s'exerçant au profit d'associations sélectionnées par l'entreprise. Ces activités s'effectuent pendant le temps de travail, ou sur le temps libre des salariés » (Bory, 2013, 53). De par leur appartenance affichée à un groupe, les «salariés-bénévoles » (comme les nomme Bory) participent ainsi à la promotion de l'image de marque de leur employeur. Dans une perspective très utilitaire, le bénévolat d'entreprise représente « un outil destiné à servir des objectifs de productivité, de réputation interne et externe et de rationalisation du mécénat »(Bory, 2013, 56). On se situe donc essentiellement ici dans le registre de la motivation extrinsèque.

Pour illustrer le lien que nous établissons entre le bénévolat d'entreprise et l'AVE-In, nous nous permettons de reprendre ici les paroles d'un interviewé recueilli lors de notre enquête de 2015-2016 réalisée auprès d'une vingtaine de recruteurs et de consultants RH canadiens et français :

Au niveau du profil LinkedIn des gens on demandait, ce n'était pas une obligation, mais c'était notre quotte sociale si je puis dire entre guillemets, on demandait aux gens de mettre un lien vers cet organisme à but non lucratif pour le faire connaître et aider les gens qui pouvaient être intéressés par sa mission. On faisait office de porte-parole de cette organisation-là qui était je le répète sans but lucratif à mission sociale, mais ça demeurait quand même sur une base volontaire au niveau des gens de notre organisation. (entretien 16 bis)

Trois critères majeurs du bénévolat d'entreprise sont palpables au travers de cette citation d'entretien: 1) le caractère a priori choisi d'une activité qui fait l'objet d'une incitation de la part de l'employeur, 2) l'intérêt social, 3) la promotion de l'image de marque de l'entreprise. Une piste intéressante nous permettant d'opérer un rapprochement entre l'AVE-In et le bénévolat d'entreprise se dessine donc ici. 


\section{Hypothèses : l'AVE-In, entre bénévolat marginal et d'entreprise}

À partir de ces différents éléments de définitions, nous avons synthétisé les différents critères définitionnels retenus (tableau 1). Dans ce tableau, nous avons utilisé le terme « instigateur » pour désigner l'individu ou l'instance décidant du contenu de l'activité. L'« actant » correspond, quant à lui, à l'individu qui s'engage dans l'activité.

Tableau 1. Synthèse du paysage définitionnel

\begin{tabular}{|c|c|c|c|c|c|}
\hline & $\begin{array}{c}\text { Liberté de } \\
\text { l'actant }\end{array}$ & $\begin{array}{l}\text { Instigateur } \\
\text { de l'action }\end{array}$ & $\begin{array}{c}\text { Plaisir } \\
\text { procuré par } \\
\text { l'activité }\end{array}$ & $\begin{array}{l}\text { Motivation } \\
\text { de l'actant* }\end{array}$ & $\begin{array}{l}\text { Gratification } \\
\text { de l'activité }\end{array}$ \\
\hline Loisir & Libre & Individu & + & $\mathrm{MI}$ & Gratuit \\
\hline Travail & Contraint & $\begin{array}{l}\text { Employeur } \\
\text { et/ou } \\
\text { individu }\end{array}$ & + et/ou $-* *$ & ME (et MI) & $\begin{array}{l}\text { Rémunéré/ } \\
\text { Gratuit }\end{array}$ \\
\hline $\begin{array}{l}\text { Bénévolat } \\
\text { classique }\end{array}$ & Libre & Individu & + et/ou - & MI & Gratuit \\
\hline $\begin{array}{l}\text { Bénévolat } \\
\text { marginal }\end{array}$ & Contraint & Individu & + et/ou - & ME & Gratuit \\
\hline $\begin{array}{l}\text { Bénévolat } \\
\text { d'entreprise }\end{array}$ & Libre & $\begin{array}{l}\text { Employeur } \\
\text { et individu }\end{array}$ & + et/ou - & $\begin{array}{c}\text { MI et/ou } \\
\text { ME }\end{array}$ & Rémunéré \\
\hline
\end{tabular}

Ici, tout nous porte à croire que l'AVE-In se situe à la lisière entre le bénévolat d'entreprise et un bénévolat marginal sachant d'une part qu'il favorise l'image de marque d'un employeur et, d'autre part, que la frontière entre le sentiment d'obligation et de liberté nous semble pouvoir être aisément franchie lorsque l'on fait face à l'«incitation» d'un employeur ou lorsque des formations à l'utilisation de LinkedIn sont dispensées en entreprise (Hoblingre Klein et al., 2016). Une recruteuse témoigne :

Vous pouvez, en tant qu'employeur inciter à alimenter des groupes de discussion, pour faire parler de l'entreprise, ça ne me semble pas incohérent dans la mesure où c'est sur la base d'un volontariat, et ça vous permet de maitriser en tant qu'employeur votre marque (entretien 9).

Le motif de l'incitation, qui laisse toute la place au libre-choix de l'individu, ne permet-il pas à l'employeur de s'immiscer sur un territoire privé : celui du profil LinkedIn de son employé ? L'impact commercial et financier de l'AVE-In est-il camouflé derrière une idéologie de la participation, d'engagement pour le bien commun ? Les valeurs associées à l'acte bénévole sont-elles réutilisées par les entreprises pour favoriser le développement d'activité d'AVE-In par leurs salariés ?

En tout état de cause, tout se passe comme si l'AVE-In se situait à la lisière entre le bénévolat d'entreprise et le bénévolat marginal et comme s'il s'agissait d'une extension bénévole de l'activité salariée. Une soushypothèse renforce encore ce présupposé : celle que l'AVE-In coïncide d'un point de vue temporel avec le travail salarié. En effet, les graphiques générés par l'outil Google Trends (tableau 2) nous ont permis d'observer la fréquence à laquelle le mot clef «LinkedIn » était saisi dans Google en France entre août 2015 et août 2016. Une analyse détaillée de ce graphique nous a poussés à établir un recoupement entre les baisses d'activité de recherche et les temps de vacances (figure 1). Sur les figures 1 et 2, l'axe vertical indique la proportion de recherches par rapport aux taux les plus élevés qui correspondent toujours à la valeur $100^{3}$. Tout nous porte donc à croire que l'utilisation de LinkedIn coïncide avec les périodes de travail contrairement à Facebook, puisque le mot «Facebook » est googlisé de manière relativement homogène dans le temps, indépendamment du calendrier des congés et des jours fériés (figure 2).

3. Par exemple, « une valeur de 50 signifie que le mot clef a été utilisé moitié moins souvent [...] et une valeur de 0 correspond à une région ayant enregistré moins de $1 \%$ de correspondances, par rapport à celle qui a obtenu 100 » (Google Trends). 
Certes, l'activité des individus sur LinkedIn ne peut être réduite à la recherche d'un mot clef, car il ne faut pas confondre "une mesure d'activité » avec « une mesure du phénomène sur lequel s'exerce cette activité » (Cardon, 2015, 43). Toutefois, les données de Google Trends donnent une indication importante sur la manière dont l'intérêt pour LinkedIn est associé au temps du travail.

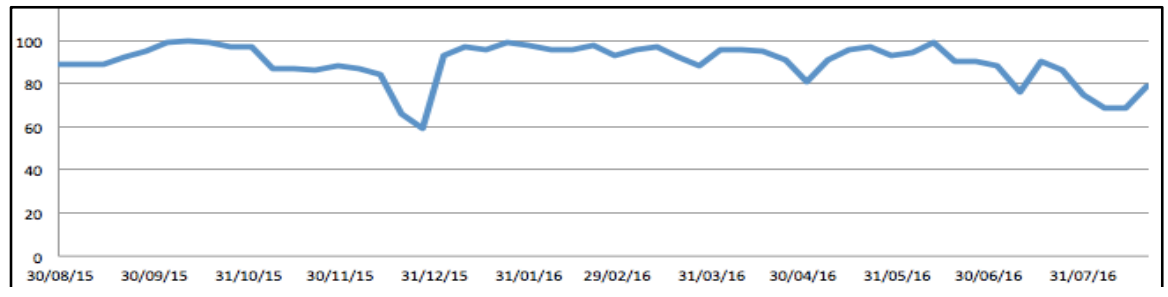

Figure 1. Évolution de l'intérêt pour la recherche du mot clef "LinkedIn" sur Google en France d'août 2015 à août 2016

Tableau 2. Diminution de la recherche du mot clef "LinkedIn" dans Google coïncidant avec les congés - analyse du schéma 14

\begin{tabular}{|c|c|c|}
\hline Dates & $\begin{array}{c}\text { Type de } \\
\text { diminution }\end{array}$ & Événement explicatif \\
\hline 16 août - 22 août 2015 & D2 & 15 août férié \\
\hline 27 décembre 2015 - 2 janvier 2016 & D3 & 25 décembre/1er janvier fériés \\
\hline $1^{\text {er }}$ mai - 7 mai 2016 & D1 & 14 juillet férié \\
\hline 10 juillet - 16 juillet 2016 & D1 mai fériés \\
\hline 31 juillet - 13 août 2016 & D1 & Période de vacances scolaires \\
\hline \multicolumn{2}{|c|}{ D3 : Diminution la plus importante, entre 55 et 65} \\
D2 : Diminution très importante, entre 65 et 75 \\
D1 : Diminution importante, entre 75 et 80 \\
\hline
\end{tabular}

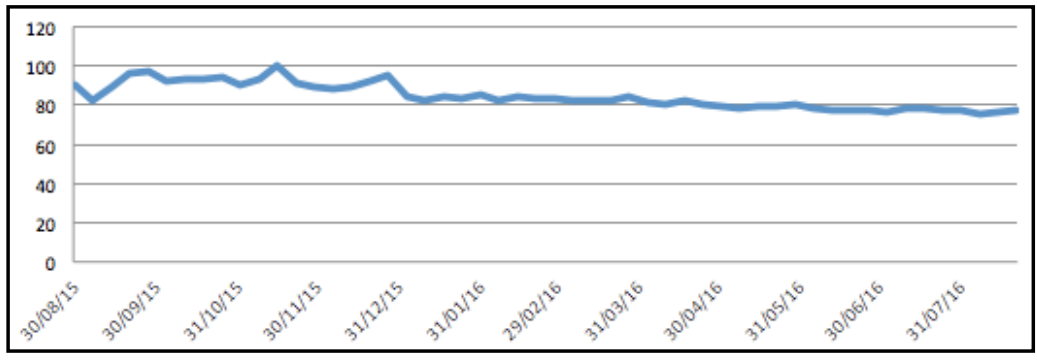

Figure 2. Évolution de l'intérêt pour la recherche du mot clef "Facebook" sur Google en France d'août 2015 à août 2016

4. Données extraites de Google Trends. 


\section{Méthodologie}

\subsection{Le choix du questionnaire comme outil d'enquête}

L'objectif général de notre enquête était d'appréhender les critères associés à l'AVE-In ainsi qu'un faisceau d'indices (au travers des représentations et des conditions d'exercice de l'AVE-In) afin de vérifier notre l'hypothèse. Pour ce faire, nous avons opté pour une analyse exclusivement quantitative par le biais d'un questionnaire. Trois raisons majeures sous-tendent ce choix.

Premièrement, nous souhaitions cerner les pratiques effectives des utilisateurs de LinkedIn ainsi que leurs représentations et leurs opinions 5 . Deuxièmement, il était essentiel d'étendre au maximum notre échantillon afin de pouvoir observer d'éventuelles tendances d'un secteur d'activité à un autre. Troisièmement, suite à l'enquête qualitative que nous avions réalisée, il nous semblait que l'AVE-In pouvait tout aussi bien être perçue comme une activité libre, volontaire, contrainte ou suggérée en fonction de la subjectivité de chacun et non de ses conditions effectives d'utilisation. Stebbins prend ainsi l'exemple d'une personne qui accepterait d'organiser le pique-nique d'une entreprise : «Si cette activité constitue un véritable acte bénévole ou un véritable loisir : tout dépend de la manière dont l'individu la définit $»^{6}$ (Stebbins, 1996, 220). En dernier lieu, notre enquête possédait trois enjeux : un enjeu descriptif (comment l'AVE-In est-il pratiqué ?), un enjeu explicatif (pour quelles raisons l'AVE-In est-il pratiqué ?) et enfin un enjeu de vérification de l'hypothèse formulée (l'AVE-In se situe-t-il à la lisière entre bénévolat marginal et d'entreprise), trois catégories de questions auxquelles les questionnaires permettent justement de répondre (Vilatte, 2007).

\subsection{Objectif et articulation du questionnaire}

Sachant que le loisir, le travail et les différents types de bénévolat énumérés plus haut possèdent de nombreux points communs, nous avons accordé une place toute particulière aux représentations et aux opinions des répondants, c'est-à-dire à leur subjectivité.

Pour appréhender au mieux les caractéristiques communes entre l'AVE-In et le bénévolat d'entreprise, il était nécessaire de comprendre comment l'AVE-In s'intégrait au cadre du travail salarié, le dépassait ou le complétait. Nous avons également tenté d'identifier le niveau de contrainte poussant l'individu à s'engager publiquement sur LinkedIn au nom d'un employeur. Notre objectif était de cerner dans quelles mesures l'AVE-In constituait une activité libre ou contrainte. C'est cette part de liberté qui devait nous permettre le cas échéant de qualifier cette activité de bénévolat classique ou de bénévolat marginal. Notre questionnaire visait enfin à identifier les récompenses et les gratifications symboliques que tirent les utilisateurs de l'AVEIn, afin d'appréhender son niveau d'instrumentalisation à l'instar du bénévolat marginal.

5. Il s'agit cependant d'être précautionneux sur ce point car «le questionnaire porte sur ce que les gens disent, sur du déclaratif et non sur ce qu'ils font effectivement. Il peut [donc] y avoir décalage, différence entre les deux » (Vilatte, 2007).

6. Traduction de l'auteur: Whether activity of this sort is true volunteering and true leisure depends on how he or she defines it (Stebbins, 1996, 220). 


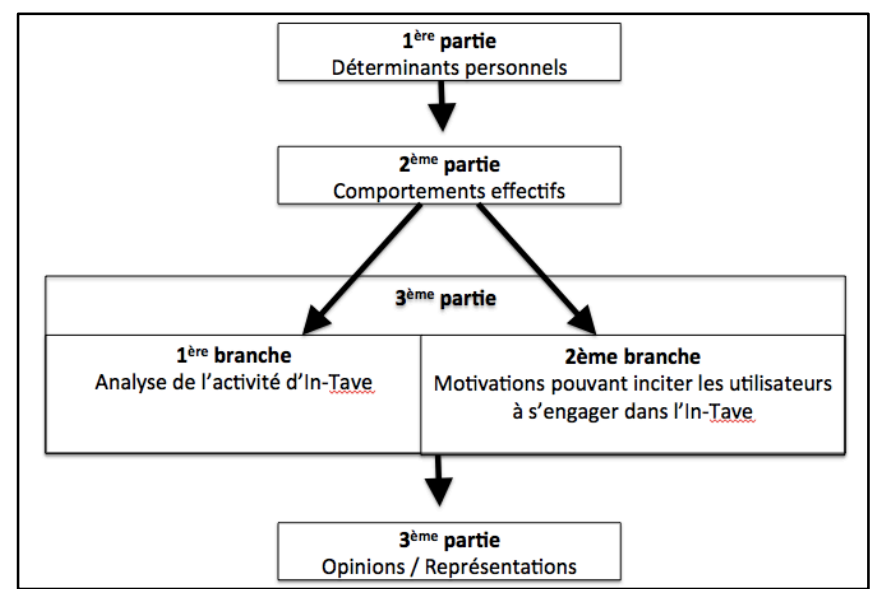

Figure 3. Structure du questionnaire d'enquête

Tableau 3. Questions phares du questionnaire - liste non exbaustive

\begin{tabular}{|c|c|}
\hline Thème & Question \\
\hline \multirow{2}{*}{ La gratuité } & $\begin{array}{l}\text { Seriez-vous prêt à faire gratuitement la promotion de votre employeur } \\
\text { sur LinkedIn via votre profil ? }\end{array}$ \\
\hline & $\begin{array}{l}\text { Valoriser sur LinkedIn l'entreprise pour laquelle vous travaillez, est-ce } \\
\text { que cela sort pour vous du cadre de votre travail salarié ? }\end{array}$ \\
\hline \multirow{3}{*}{ La liberté } & $\begin{array}{l}\text { Un employeur vous a-t-il déjà demandé de le mentionner sur votre } \\
\text { profil Linkedln? }\end{array}$ \\
\hline & $\begin{array}{l}\text { Votre utilisation de Linkedln est-elle imposée par l'entreprise qui vous } \\
\text { emploie? }\end{array}$ \\
\hline & $\begin{array}{l}\text { Un employeur vous a-t-il déjà demandé d'envoyer des posts sur } \\
\text { Linkedln pour transmettre des informations le concernant? }\end{array}$ \\
\hline \multirow[t]{2}{*}{ L'engagement } & $\begin{array}{l}\text { Défendre des valeurs ou une cause commune, vous engager } \\
\text { socialement, est-ce que cela pourrait vous pousser à mettre en avant } \\
\text { votre employeur sur votre profil Linkedln? }\end{array}$ \\
\hline & $\begin{array}{l}\text { Considérez-vous que votre profil Linkedln doive traduire votre } \\
\text { engagement par rapport à l'entreprise pour laquelle vous travaillez? }\end{array}$ \\
\hline Travail et loisir & $\begin{array}{l}\text { Valoriser l'image de son employeur par le biais de son profil Linkedln en } \\
\text { ajoutant une description de l'entreprise, considérez-vous cela comme } \\
\text { un travail, un loisir ou ni l'un ni l'autre? }\end{array}$ \\
\hline
\end{tabular}

Suivant ce cahier des charges, le questionnaire s'est articulé en cinq parties (figure 3). La première portait sur les déterminants personnels et la deuxième sur l'identification des comportements effectifs. À partir des réponses données dans la deuxième partie, la troisième partie se divisait en deux branches suivant le principe des questions à tiroir. La première branche était destinée aux individus pratiquant l'AVE-In et permettait une analyse de cette activité. La deuxième branche était destinée aux individus ne pratiquant pas l'AVE-In et permettait d'identifier les motivations pouvant pousser les personnes à s'engager dans ces activités. Enfin, la quatrième partie du questionnaire était focalisée sur les opinions et les représentations des tous les répondants.

Au travers de ce questionnaire nous souhaitions aborder sept thèmes importants : la gratuité, la liberté, le travail salarié, l'engagement (notamment social), les valeurs (celles de l'individu vs celles de l'employeur), 
le travail et le loisir. En fonction de ces thèmes, nous avons formulé trente questions dont certaines (tableau 3) sont particulièrement emblématiques de cette recherche.

Les questions ont souvent été formulées sous forme de tableaux à compléter, tels que le tableau 4 afin de différencier les trois activités désignées par le sigle AVE-In.

Tableau 4. Exemple de question présentée sous forme d'un tableau

\begin{tabular}{|l|c|c|c|}
\hline \multicolumn{2}{|c|}{ A quel moment vous consacrez-vous à (aux) l'activité(s) qui vous concernent ? (cochez) } \\
\hline & $\begin{array}{c}\text { Utilisation de votre } \\
\text { profil comme } \\
\text { outil de travail }\end{array}$ & $\begin{array}{c}\text { Descriptif de } \\
\text { I'entreprise ajouté } \\
\text { à votre profil }\end{array}$ & $\begin{array}{c}\text { Envoi de posts } \\
\text { depuis votre profil }\end{array}$ \\
\hline $\begin{array}{l}\text { Pendant vos } \\
\text { horaires de travail }\end{array}$ & & & \\
\hline $\begin{array}{l}\text { Hors de vos } \\
\text { horaires de travail }\end{array}$ & & & \\
\hline
\end{tabular}

\section{Résultats}

Les différentes catégories de répondants seront désignées sous la forme de sigles (tableau 5). En ce qui concerne la conversion du nombre de réponses en pourcentages, elle a été établie selon des totaux spécifiques en fonction des parties du questionnaire correspondantes. Par exemple, 11 oui apparaissant dans la branche 1 seront calculés selon la formule suivante : 11 x 100/72 = 15,27\%

Tableau 5. Désignation des différents répondants par abréviations et calcul des pourcentages

\begin{tabular}{|l|l|l|l|}
\hline Type de répondants & Sigle & Total & Total utilisé pour les calculs relatifs à \\
\hline Tous les répondants & RT & 117 & Parties 1, 2 et 4 \\
\hline Répondants de la branche 1 & R1 & 72 & Branche 1 \\
\hline Répondants de la branche 2 & R2 & 45 & Branche 2 \\
\hline
\end{tabular}

\subsection{Analyse de l'activité d'AVE-In chę les R1}

Chez les R1 l'utilisation de LinkedIn comme outil de travail (56\% des R1) est presque autant représentée que l'ajout d'un descriptif relatif à son employeur sur son profil (54\% des R1). En revanche, l'envoi de posts reste plus occasionnel (31\% des R1). Il convient donc de veiller à différencier ces trois activités pour une appréhension exacte de l'AVE-In.

La majorité des R1 consacre moins d'une heure par semaine à l'AVE-In. Lorsqu'on dépasse cette durée, c'est l'utilisation du profil comme outil de travail qui est la plus représentée (figure 4).

L'utilisation du profil comme outil de travail est davantage pratiquée sur le temps de travail (figure 5) comparativement aux deux autres activités. Pourtant, elle reste globalement perçue comme librement choisie (tableau 6). On constate par ailleurs que le moment consacré à l'AVE-In hors-travail reste très stable d'une activité à une autre (figure 5), ce qui peut nous permettre d'y voir l'expression de l'appropriation de LinkedIn par ses utilisateurs, sachant que « la planification du temps est un facteur déterminant dans l'adoption d'une nouvelle technologie » (Trestini et al., 2016, 186). 


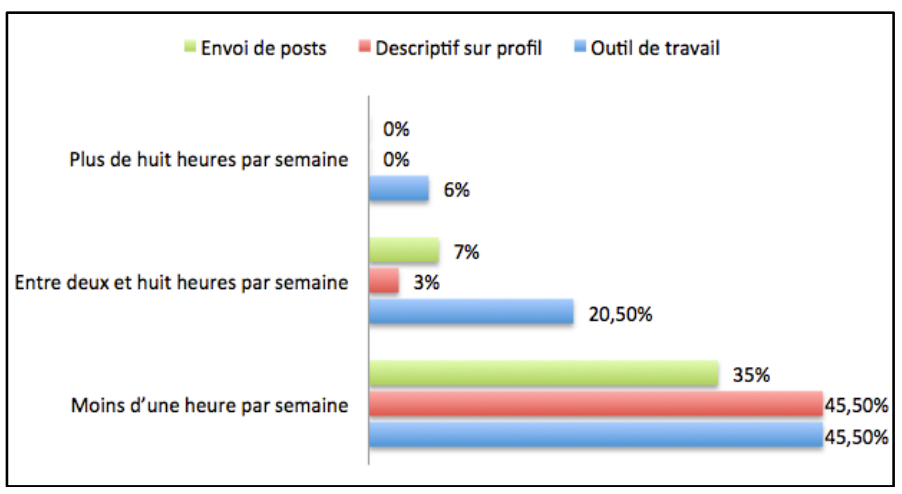

Figure 4. Durée consacrée aux trois activités d'AVE-In chez les R1

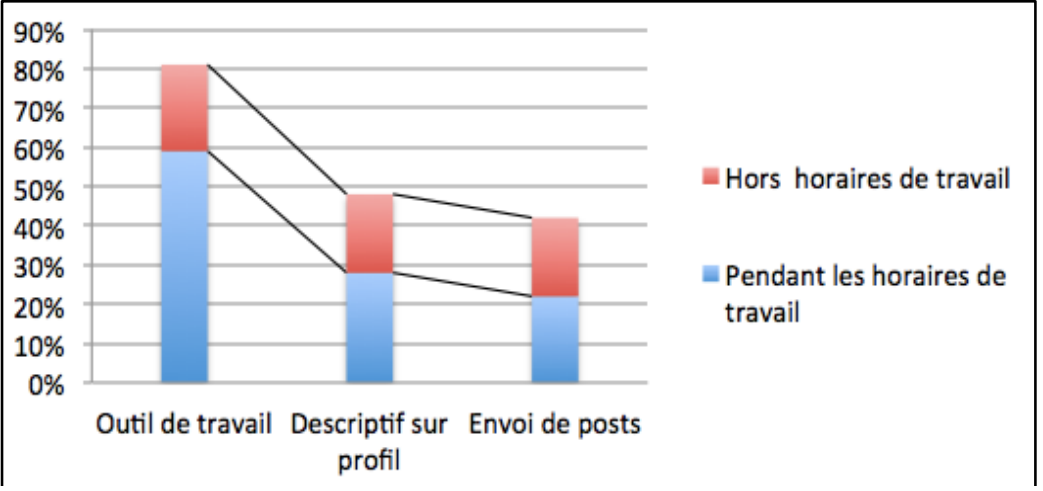

Figure 5. Moment consacré aux 3 activités d'AVE-In chez les R1

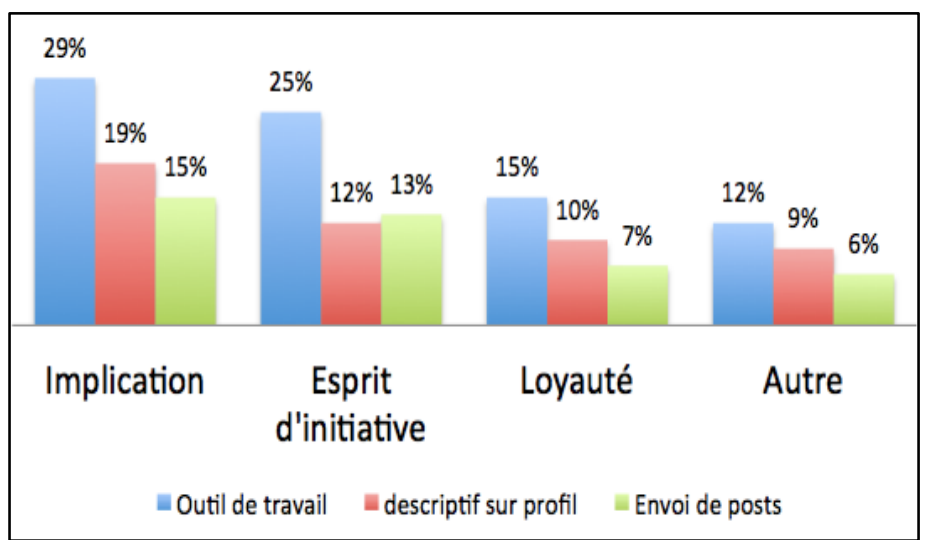

Figure 6. Signal que l'individu soubaite renvoyer à son employeur au travers de son activité d'AVE-In 
Quant aux motivations pouvant pousser les utilisateurs à pratiquer l'AVE-In, on compte surtout le désir d'envoyer un signal d'implication à son employeur et de faire montre d'esprit d'initiative (figure 6) : on se situe donc surtout dans le registre de la motivation extrinsèque. On remarque de surcroît que $60 \%$ des R1 considèrent que l'utilisation de leur profil comme outil de travail peut leur permettre d'asseoir leur identité professionnelle. Sur cette base, on peut faire l'hypothèse que la motivation des individus pratiquant l'AveIn est avant tout liée à l'image de soi, ce qui serait assez logique sachant qu'un « engagement motivé par l'image de soi va favoriser des activités plus visibles » (Bougard et al., 2013, 49). Or, les activités d'Ave-In trouvent toute leur force dans leur visibilité.

\subsection{Motivations pouvant pousser les $\mathrm{R} 2$ à s'engager dans l'AVE-In}

Quant aux motivations pouvant pousser les R2 à s'engager dans l'AVE-IN, près d'un quart d'entre eux seraient prêts à faire gratuitement la promotion de leur employeur sur leur profil LinkedIn et presque autant réclameraient une rémunération. Ceci signifie que la moitié a préféré ne pas répondre à cette question, une abstention qui indique certainement une indétermination quant à la question de la rémunération, d'autant plus que les pourcentages des deux autres réponses $(24,4 \%$ et $26,7 \%)$ sont assez proches.

\subsection{Opinions/représentations des RT}

En ce qui concerne les opinions et les représentations, $54 \%$ des RT ont affirmé que défendre des valeurs ou une cause commune, s'engager socialement (motivations qui rappellent celles des bénévoles «classiques ») pourrait les pousser à mettre en avant leur employeur sur leur profil. À partir de ce dernier constat, nous nous demandons si le partage de valeurs communes entre employeur et employé pourrait inciter les employés à s'investir dans des activités bénévoles de valorisation de l'image de l'entreprise.

Par ailleurs, valoriser l'image de son employeur en publiant des informations sur son profil LinkedIn est perçu essentiellement comme un travail (40\% des RT) ou comme une activité ne constituant ni un travail ni un loisir (46\% des RT), mais les répondants repoussent l'idée selon laquelle l'AVE-In pourrait constituer un loisir : seuls $3 \%$ des RT catégorisent l'AVE-In comme loisir. La nécessité du recours à une nouvelle terminologie pour catégoriser cette activité se fait donc sentir ici.

Tableau 6. Sentiment de liberté ou de contrainte dans l'exercice de l'AVE-In chez, les R1

\begin{tabular}{|l|c|c|c|}
\hline & $\begin{array}{c}\text { Imposée } \\
\text { par } \\
\text { l'entreprise }\end{array}$ & $\begin{array}{c}\text { librement choisie : } \\
\text { personne ne vous } \\
\text { a demandé ou } \\
\text { suggéré d'y être }\end{array}$ & $\begin{array}{c}\text { à la fois libre et imposée : } \\
\text { vous avez librement choisi } \\
\text { d'utiliser un réseau } \\
\text { incontournable }\end{array}$ \\
\hline Outil de travail & $7 \%$ & $35 \%$ & $25 \%$ \\
\hline $\begin{array}{l}\text { Descriptif sur } \\
\text { profil }\end{array}$ & $10 \%$ & $31 \%$ & $13 \%$ \\
\hline Envoi de posts & $6 \%$ & $21 \%$ & $18 \%$ \\
\hline
\end{tabular}

Enfin, près des trois-quarts des R2 considèrent que valoriser sur LinkedIn l'entreprise pour laquelle ils travaillent sortirait du cadre de leur travail salarié. Ici, sachant que le travail salarié est placé sous le signe de la contrainte extérieure (puisqu'un lien de subordination unit l'employé à son employeur), il est assez remarquable que l'AVE-In soit perçue, par ceux qui la pratiquent, comme une activité librement choisie (tableau 6). Ceci rapproche alors l'AVE-In du bénévolat si l'on s'en réfère à cette citation de Ferrand: «Acteur non contraint, le bénévole est libre. Rien, sauf l'honneur, n’oblige à faire du bénévolat » (FerrandBechmann, 2000, 109).

À partir de ces différents résultats, nous avons mesuré le niveau de correspondance globale entre les caractéristiques des cinq catégories d'activité mises en exergue plus haut (tableau 1) et celles de l'Ave-In (tableau 7). Pour ce faire, nous avons identifié quatre niveaux de caractérisation : fort, moyen, faible et à vérifier. En cas de question à choix multiple, une caractéristique forte correspond à la réponse la plus 
représentée, pourvu qu'il y ait un écart d'au-moins $10 \%$ par rapport à aux autres réponses proposées. En cas de question fermée, un taux de réponse entre 0 et $24 \%$ est considéré comme faible, entre $25 \%$ et $50 \%$ comme moyen et entre $51 \%$ et $100 \%$ comme fort. Les caractéristiques à vérifier correspondent à celles ne donnant pas lieu à des résultats suffisamment parlants : c'est le cas notamment lorsque de trop faibles écarts de réponse sont observés.

Tableau 7. Positionnement de l'Ave-In dans le paysage définitionnel retenu

\begin{tabular}{|l|c|c|c|c|c|}
\hline & $\begin{array}{c}\text { Liberté de } \\
\text { I'actant }\end{array}$ & $\begin{array}{c}\text { Instigateur } \\
\text { de l'action }\end{array}$ & $\begin{array}{c}\text { Plaisir } \\
\text { procuré par } \\
\text { I'activité }\end{array}$ & $\begin{array}{c}\text { Motivation } \\
\text { de l'actant }\end{array}$ & $\begin{array}{c}\text { Gratification } \\
\text { de l'activité }\end{array}$ \\
\hline Loisir & Libre & Individu & + & $\mathrm{MI}$ & Gratuit \\
\hline Travail & Contraint & $\begin{array}{c}\text { Employeur } \\
\text { et/ou individu }\end{array}$ & + et/ou - & $\mathrm{ME}$ (et MI) & $\begin{array}{c}\text { Rémunéré / } \\
\text { Gratuit }\end{array}$ \\
\hline $\begin{array}{l}\text { Bénévolat } \\
\text { classique }\end{array}$ & Libre & Individu & + et/ou - & $\mathrm{MI}$ & Gratuit \\
\hline $\begin{array}{l}\text { Bénévolat } \\
\text { marginal }\end{array}$ & Contraint & Individu & + et/ou - & $\mathrm{ME}$ & Gratuit \\
\hline $\begin{array}{l}\text { Bénvolat } \\
\text { d'entreprise }\end{array}$ & Libre & $\begin{array}{c}\text { Employeur et } \\
\text { individu }\end{array}$ & + et/ou - & $\begin{array}{c}\mathrm{MI} \text { et/ou } \\
\mathrm{ME}\end{array}$ & Rémunéré \\
\hline
\end{tabular}

\section{Légende}

Ceci nous a permis de rapprocher l'Ave-In du loisir, du bénévolat classique et du bénévolat marginal. Il serait nécessaire, lors d'une étude ultérieure, de comprendre le niveau de plaisir ou de déplaisir procuré par cette activité, et de cerner davantage la part des motivations intrinsèques par rapport aux motivations extrinsèques mises en évidence. De plus, il faut bien noter que les résultats concernant la gratuité ou la rémunération de cette activité sont trop mitigés pour nous permettre de distinguer une quelconque tendance.

\section{Discussion}

\subsection{Déconnexion et extension bénévole de l'activité salariée (1 ligne) ???}

Comme nous l'avons remarqué plus haut, le moment consacré à l'AVE-In hors-travail reste le même quelle que soit l'activité pratiquée. On peut alors se demander si cela ne reflète pas un débordement du travail sur le temps de loisir, à moins qu'il ne s'agisse d'une extension bénévole de l'activité salariée, ou encore d'une problématique de déconnection. En effet, c'est en premier lieu le désir d'être intégré et reconnu qui pousse l'individu à rester connecté (Jauréguiberry, 2014). Or, l'AVE-In s'impose comme un moyen pour les individus de renvoyer un signal de loyauté et d'implication, deux notions relevant d'une logique d'appartenance. Enfin, avec l'apparition des nouveaux outils de communication, «l'urgence professionnelle fait irruption dans l'espace privé en le "trouant" sous la forme d'astreintes, de gardes ou de parenthèses téléphoniques » (Jauréguiberry, 2014, 25) : l'activité d'AVE-In constituerait-elle une astreinte continue, sur une plateforme où les profils incarnent à la fois des espaces professionnels et des espaces plus " personnels » de pilotage de carrière (Hoblingre Klein et al., 2016)?

\subsection{Communiquer ou promouvoir}

Les réponses obtenues ne nous permettent pas d'affirmer que l'AVE-In constitue une activité bénévole, même si le lien avec le bénévolat reste tangible. Nous nous demandons si cette difficulté ne résulte pas d'un questionnement irrésolu : celui de la délimitation entre les activités journalistiques et publicitaires. On a ainsi vu se développer sur internet le native advertising décrit comme suit par Carlson « les publicitaires créent ou sponsorisent un contenu qui tend à se confondre avec un contenu éditorial $»^{7}($ Carlson, 2015, 2). Toute

7. Traduction de l'auteur de : advertisers create or sponsor content intended to blend in with the editorial content (Carlson, 2015, 2). 
l'ambivalence du native advertising se situe donc dans le clivage entre recherche de profit économique et volonté de servir la communauté. Dans un contexte où les publicités natives adoptent l'allure d'articles journalistiques à caractère informatif, on peut se demander quelles sont les motivations prioritaires des individus pratiquant l'AVE-In. Souhaitent-ils avant tout promouvoir leur employeur ou bien renseigner les internautes ? La communication marketing prévaut-elle sur l'information ? La réflexion de ce consultant en ressources humaines interviewé lors d'une précédente enquête illustre l'ambiguité de l'AVE-In, entre activité informationnelle et marketing :

"En ce moment nous créons une marque : je le mets aussi sur LinkedIn, ça peut intéresser ou non les gens, c'est aussi de la communication.» [entretien 1]

Déterminer le caractère informationnel de l'AVE-In constitue sans doute une condition nécessaire pour nous aider à comprendre s'il peut s'agir d'une sorte de bénévolat. En effet, l'activité publicitaire nous semble relativement peu tournée vers le bien commun tandis qu'informer nous semble davantage pouvoir correspondre aux motivations des bénévoles « classiques ", à l'instar des blogueurs passionnés par un sujet de pointe et des pionniers de l'internet.

\subsection{Vers un paternalisme numérique propice au bénévolat?}

Les résultats de notre questionnaire démontrent l'intérêt identitaire de l'AVE-In et le signal d'implication que les individus souhaitent renvoyer au travers de ces activités. La fusion identitaire employé/employeur palpable au travers de certains profils pourrait quant à elle être interprétée comme une marque d'attachement affectif, d'autant plus qu'une majorité de R2 s'accorde à dire que le partage de valeurs communes avec leur employeur pourrait les inciter à s'investir dans des activités de valorisation de son image sur LinkedIn. Par là même, l'AVE-In n'est-il pas l'expression d'une fusion identitaire employé/employeur que l'on peut rapprocher d'un certain paternalisme numérique? Ce nouveau concept que nous proposons d'introduire serait alors défini comme le contrôle partiel exercé par l'entreprise sur la vie numérique de ses employés, avec le consentement implicite de ces derniers. Ce paternalisme numérique nous semble pouvoir favoriser les activités bénévoles de par le brouillage des frontières entre la sphère privée et professionnelle qu'il occasionne. L'espace d'engagement du salarié serait alors élargi et favoriserait dans le même temps l'élargissement de l'espace de contrôle de l'employeur (jusque dans le profil LinkedIn de ses employés) et le consentement des individus face à ce contrôle.

\section{Conclusion - ouverture}

Les résultats du questionnaire ne nous permettent pas d'affirmer que l'AVE-In constitue une activité bénévole, même si le lien avec le bénévolat reste tangible. Cependant, ils démontrent que la catégorisation binaire travail-loisir doit être dépassée pour caractériser l'AVE-In. Ils témoignent également de représentations et de pratiques différentes en ce qui a trait aux trois activités désignées par le sigle AVE-In : l'envoi de posts, l'utilisation de LinkedIn comme outil de travail et la valorisation de l'employeur sur le profil. Pour une étude ultérieure, il conviendrait sans doute de se concentrer sur une seule de ces trois activités, d'autant plus que la critique que nous pouvons adresser à notre questionnaire est sa complexité.

En effet les questions ont souvent été formulées sous la forme de tableaux (tableau 4) ce qui a peut-être généré des erreurs dans les résultats. Des écarts incompréhensibles entre certaines réponses peuvent en tout cas nous amener à le croire. Ainsi, le nombre d'individus déclarant, au tout début du questionnaire, utiliser LinkedIn comme outil de travail ne coïncide pas toujours exactement avec le nombre de réponses relatives à cette utilisation dans la suite du questionnaire. Cependant, des tendances nettes et cohérentes se sont dessinées, par exemple le caractère marginal de l'utilisation de LinkedIn pour l'envoi de posts, tant en termes de nombre d'individus concernés que de temps consacré à cette activité. Les résultats, bien que contestables, nous révèlent bien une certaine réalité.

Par ailleurs, nous souhaitons souligner que les marges d'amélioration de notre questionnaire témoignent sans doute du caractère inexploré d'un terrain que notre enquête a davantage permis de défricher que d'analyser en détail. En effet, bien plus qu'une nouvelle terminologie, l'AVE-In pourrait représenter un nouveau champ disciplinaire qui se situerait à l'intersection entre celui des transformations professionnelles induites par les nouveaux outils de communication numérique et celui du Digital Labor. Les questions en lien avec les transformations professionnelles induites par les nouveaux outils de communication numérique ont été notamment abordées à travers trois thèmes majeurs : la déconnexion (Jauréguiberry, 2014), l'usage TIC 
au travail (Boudokhane-Lima \& Felio, 2015 ; Felio, 2013) et les salariés ambassadeurs 2.0 (Larroche, 2014). Ces trois thèmes sont apparus en filigrane dans l'analyse des entretiens cités dans cet article et des résultats du questionnaire. Quant au Digital Labor, l'AVE-In reprend certaines de ses problématiques : la difficile séparation entre public et privé (Cardon \& Casilli, 2015), l'extension du domaine du travail (Casilli, 2015) et la rémunération des nouvelles formes de travail qui ne disent pas leur nom. Ici, l'intérêt de voir en l'AVEIn un nouveau champ disciplinaire est triple :

- cela permettrait de circonscrire l'analyse des nouvelles formes de travail à un seul type d'espace particulier ayant ses spécificités techniques propres : les réseaux sociaux numériques professionnels.

- l'AVE-In affine la question de la perméabilité entre privé et public à celle de la distinction entre espace externe et espace interne, puisque comme le soulignent Girard, Fallery et Rodhain, LinkedIn (et a fortiori les autres RSNP tels que Viadeo et Xing) se situe à la frontière entre l'interne et l'externe (Girard et al., 2011). L'espace interne, caractérisé ici par une utilisation dans le cadre d'un emploi, engage à la fois l'individu et un système organisationnel (l'entreprise). Il s'oppose en cela à l'espace externe qui reste du seul ressort de l'individu.

- l'AVE-In nous semble signaler un changement de paradigme dans la relation employé/employeur qu'il serait sans doute bon d'analyser. En effet, on observe aujourd'hui d'une part un phénomène d'ubérisation, lequel « constitue une forme de désintermédiation-réintermédiation » (Lambrecht, 2016, 5), et, d'autre part, une extension du modèle entrepreneurial jusqu'en entreprise, alors que celui-ci a été initialement pensé comme une forme de désinstitution-nalisation (Deroy, 2008). Ces différents mouvements indiquent une mutation du salariat classique vers une forme moins réglementée en même temps qu'une mutualisation des ressources entre employés et employeurs. Ce dernier constat n'est pas sans rappeler la manière dont les communautés de pratiques sont aujourd'hui l'objet d'un processus d'institutionnalisation de la part des entreprises qui les soutiennent, un processus qui s'accompagne d'une forte tendance à formaliser de l'informel (Nezet, 2017). Cette institutionnalisation signalerait peut-être la volonté qu'ont les organisations de contrôler les informations véhiculées à leur sujet, et de s'immiscer dans un bouche-à-oreille qui, comme le signalent Philippe Viallon et Marc Boyer, font "perd[re] en partie la maitrise de sa communication » (Viallon \& Boyer, 1994, 65) à l'émetteur. En tout état de cause, on assiste sans doute à un remaniement de la frontière entre interne et externe. Ce changement de paradigme, s'il est confirmé, peut-il favoriser le déploiement d'une idéologie coopérative et ce faisant renforcer les valeurs propres au bénévolat ?

Enfin, dans son article sur l'intégration des réseaux sociaux en bibliothèque, Fanny George signale que parmi les motivations pouvant amener les individus à avoir recours à un réseau social pour leurs activités de lecteur on compte surtout « le partage des connaissances et la participation bénévole à un projet culturel » (Georges, 2010, 2). Ces deux motivations s'articulent toujours avec d'importantes problématiques de présentation de soi car « les réseaux sociaux en ligne ont en commun de relier les utilisateurs par leur profil personnel » (Ibid. 2). On comprend donc ici que les spécificités techniques des réseaux sociaux ne permettent pas de dissocier les activités informationnelles et communicationnelles (fussent-elles déployées bénévolement au seul service de la communauté) d'enjeux identitaires et réputationnels. L'engagement des salariés dans un cadre interne, même lorsqu'il est bénévole, doit être étudié en tenant compte des problématiques identitaires externes qu'il implique.

\section{Bibliographie}

- Audran J. (2002). La liste de diffusion électronique: un instrument de formation professionnelle? Recherche et Formation, 39, 123-141.

- Baudry B. \& Chassagnon V. (2017). L'arbitrage entre le salariat et le travail indépendant au prisme des théories de la firme. Revue de l'OFCE, (149), 167-189.

- Bory A. (2013). Le bénévolat d'entreprise en France. Travail et emploi, (133), 53-62.

- Boudokhane-Lima F., \& Felio C. (2015). Les usages professionnels des TIC: des régulations à construire. Communication et organisation, (48), 139-150.

- Bougard J., Brodaty T., Emond C., L'Horty Y., Parquet L. D., \& Petit P. (2013). Bénévolat et accès à l'emploi. Les enseignements d'une expérience contrôlée. Revue économique, 65(1), 47-69.

- Broca S. (2016). Le digital labor est-il vraiment du travail? InaGlobal. Retrieved from http://www.inaglobal.fr/numerique/article/le-digital-labor-est-il-vraiment-du-travail-8674

- Bruna B., \& Damiano F. (2009). Why without Pay? The Intrinsic Motivation between Investment and Consumption in Unpaid Labour Supply (CELPE Discussion Papers No. 111). CELPE - Centre of Labour Economics and Economic Policy, University of Salerno, Italy. 
- Bughin J., \& Chui M. (2010, December). The rise of the networked enterprise: Web 2.0 finds its payday. The McKinsey Quarterly.

- Cardon D. (2015). A quoi rêvent les algorithms : Nos vies à l'heure des big data. Seuil.

- Cardon D., \& Casilli A. (2015). Qu'est-ce que le Digital Labor? InaGlobal.

- Carlson M. (2015). When news sites go native: Redefining the advertising-editorial divide in response to native advertising. Journalism, 16(7), 849-865.

- Casilli A. (2015, September 11). Antonio Casilli: Poster sur Facebook, c'est travailler. Comment nous rémunérer? Libération, Retrieved from http://www.liberation.fr/ futurs/2015/09/11/antonio-casilli-postersur-facebook-c-est-travailler-comment-nous-remunerer_1380647

- Deroy X. (2008). L'événement entrepreneurial et le modèle entrepreneurial. Revue Francaise de Gestion, 34(187), 51-63.

- Dujarier M.-A. (2014). Le travail du consommateur. Paris, La Découverte.

- Ertzscheid O. (2013). L’homme, un document comme les autres, Human Beings as Documents. Hermès, La Revue, (53), 33-40.

- Felio C. (2013). Pratiques communicationnelles des cadres: usage intensif des TIC et enjeux psychosociaux. Université Michel de Montaigne, Bordeaux III.

- Felstiner A. (2011). Working the crowd: employment and labor law in the crowdsourcing industry. BerkelJournal of Employment and Labour Law, 32(1), 143-202.

- Ferrand-Bechmann D. (2000). Le Métier de bénévole. Paris, Anthropos.

- Georges F. (2009). Représentation de soi et identité numérique. Réseaux, 154(2), 165-193.

- Georges F. (2010). Pratiques informationnelles et identités numériques. Études de Communication, (2), 105-120.

- Girard A., Fallery B., \& Rodhain F. (2011). L’apparition des médias sociaux dans l'e-GRH: gestion de la marque employeur et e-recrutement. In Actes du congrès AIM (pp. 1-18).

- Guillaume L.-P. (2017). Communities@Work: des communautés en réseau chez Schneider Electric. In Communautés de pratique et management de la formation (Presses universitaires de l'UTBM). Belfort.

- Hoblingre Klein H. \& Audran J. (2017). Une communauté professionnelle en réseau : LinkedIn, un premier pas vers la formation? In Communautés de pratique et management de la formation (Presses universitaires de l'UTBM). Belfort.

- Hoblingre Klein H., Audran J., \& Chevry Pébayle E. (2016). Analyse de l'activité de consultants RH et de recruteurs français sur LinkedIn: entre outil de travail et quête de dé-veloppement identitaire. ESSACHESS_ Journal for Communication Studies, 9(2 (18)), 153-170.

- Hoblingre Klein H., \& Chevry Pébayle E. (2016). L’utilisation de LinkedIn en France et au Canada : une analyse comparée. In Médias numériques et communication électronique (p. 1020). Bruges: Aquaprint.

- Jacquot L. (2012). Peut-on penser le travail par le don ? Présentation du débat. La nouvelle revue du travail, (1).

- Jauréguiberry F. (2014). La déconnexion aux technologies de communication. Réseaux, (186), 15-49.

- Kesteman M. \& Monnier E. (2005). Bénévoles et rémunérés : tous professionnels ? Pensée plurielle, 9(1), 5560 .

- Lambrecht M. (2016). L'économie des plateformes collaboratives. Courrier hebdomadaire du CRISP, 2311(26), 5-80.

- Larroche V. (2014). Quelle visibilité professionnelle pour un salarié ambassadeur 2.0 auprès des recruteurs potentiels? Communication \& Organisation, (44), 53-64.

- Larroche V., \& Piment H. (2014). De l'habileté dans les interactions numériques aux compétences professionnelles de l'animateur de communautés en ligne. In Travail et loisirs Comment le travail se transforme par et dans le divertissement et comment le divertissement se requalifie en travail? (pp. 159-167).

- Mercier D. \& Plaisent M. (2010). Organisation des connaissances dans les habitats numériques de communauté. Une étude exploratoire sur un cas de communauté de pratique de bienfaisance. Les Cabiers Du Numérique, 6(3), 167-185. https://doi.org/10.3166/lcn.6.3.167-185

- Merzeau L. (2015). E-réputation : carrefour et tensions. In C. Alcantara, E-réputation : regards croisés sur une notion émergente. Issy-les-Moulineaux, Gualino Editions.

- Metzger J.-L. \& Cléach O. (2004). Le télétravail des cadres : entre suractivité et apprentissage de nouvelles temporalités. Sociologie du Travail, 46(4), 433-450.

- Nezet M. (2017). L'animation des communautés de pratique en jeu pour le développement professionnel des membres et la compétitivité de l'entreprise. In Communautés de pratique et management de la formation (Presses universitaires de l'UTBM). Belfort.

- Pierre J., \& Alloing C. (2015). Questionner le digital labor par le prisme des émotions : le capitalisme affectif comme métadispositif? In La communication numérique au coeur des sociétés: dispositifs, logiques de développement et pratiques. Echirolles, France, GRESEC.

- Povéda A. (2016). L’identité numérique des jeunes diplômés : les risques de l'absence. Études de communication, (47), 167-182.

- Riverin D. \& Simard Y. (2003). Au-delà du lien travail-loisir: la citoyenneté. Revue Internationale de Carriérologie, 9, 1-2. 
- Schwartz Y. (2010). Connaître et étudier le travail. Le Philosophoire, (2), 71-78.

- Schwartz Y. \& Durrive L. (2003). Travail et Ergologie. Entretiens sur l'activité bumaine (Première édition). Toulouse, Octares Editions.

- Simonet M. (2010). Le travail bénévole : Engagement citoyen ou travail gratuit? Paris, La Dispute.

- Stebbins R. A. (1996). Volunteering: A Serious Leisure Perspective. Nonprofit and Voluntary Sector Quarterly, 25(2), 211-224.

- Trestini M., Coulibaly B., Rossini I. \& Pébayle E. C. (2016). Appropriation sociale des MOOC en France (Sciences, société et nouvelles technologies). London, ISTE éditions.

- Valenduc G. \& Vendramin P. (2016). Le travail dans l'économie digitale : continuités et ruptures. ETUI, Institut Syndical Européen, WP.

- Vendramin P., Valenduc G., \& others. (2016). Le travail virtuel. Nouvelles formes d'emploi et de travail dans l'économie digitale (p. 41). UCL.

- Vial S. (2013). L'être et l'écran: comment le numérique change la perception. Paris, Presses Universitaires de France.

- Viallon P. \& Boyer M. (1994). La communication touristique. Paris, Presses Universitaires de France.

- Vilatte J.-C. (2007). Méthodologie de l'enquête par questionnaire. Université d'Avignon. Retrieved from http://www.lmac-mp.fr/images/imagesFCK/file/outils/jcvilatte/ jcvilatte_le_questionnaire_jcv.pdf

- Zedini C., Cheikh A. B., Limam M., Henrichi Y., Mellouli M., Ghardallou M. E., Ajmi, T. (2016). Les facteurs de motivation au travail chez les cadres paramédicaux dans un hôpital tunisien, Determinants of paramedical staff motivation in a Tunisian hospital. Santé Publique, 28(5), 613-622. 